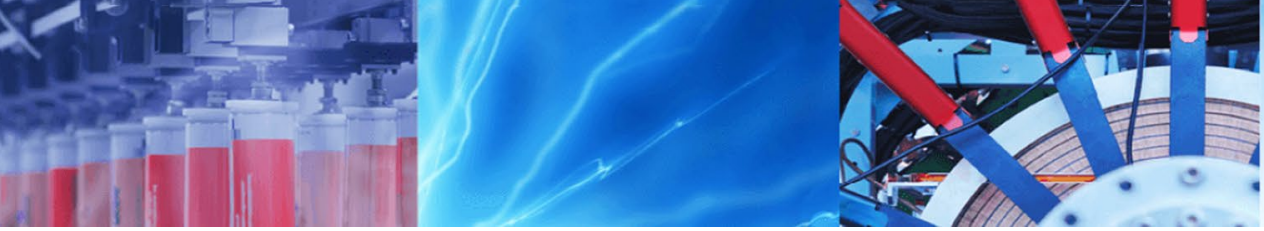

Research Article

\title{
Synthesis, characterization and Hall-effect studies of highly conductive polyaniline/graphene nanocomposites
}

\author{
T. Rajyalakshmi ${ }^{1} \cdot$ Apsar Pasha $^{2}$ (D) Syed Khasim ${ }^{1,3,4} \cdot$ Mohana Lakshmi $^{1} \cdot$ Mohammed Imran $^{5}$
}

Received: 9 December 2019 / Accepted: 26 February 2020 / Published online: 2 March 2020

(c) Springer Nature Switzerland AG 2020

\begin{abstract}
The objective of this research is to prepare a series of conducting polyaniline/graphene composites by in situ chemical oxidative method through polymerization of aniline in the existence of graphene of various weight percentages. The prepared composites were examined through Fourier transform infrared spectroscopy, X-ray diffraction and scanning electron microscopy respectively. The conductivity and dielectric attributes exhibited composition dependent percolation behavior with enhanced properties for $15 \mathrm{wt} \%$ of graphene in polyaniline matrix. The Hall-effect studies were performed in the prepared composites and the investigations confirm that the composites behave as $\mathrm{n}$-type semi-conductors. From the data obtained one can envisage remarkable improvement in the charge transport and dielectric properties of the composite at the percolation threshold due to the presence of nano-size additive in the composite. The temperature dependent conductivity in doped composites was increased by five fold in compare to pure PANI. The doping of graphene in PANI matrix, the improved dielectric parameters were achieved. The Hall effect studies were carried out in the prepared composites, the results shows that composites behaves like a n-type semiconductor. Further, the mechanical properties such as tensile stress and tensile strain were carried out in the prepared composites.
\end{abstract}

Keywords Electrical conductivity · Dielectric properties · Nanocomposites · Percolation threshold · Transport properties $\cdot$ Hall effect $\cdot$ Tensile stress $\cdot$ Tensile strain

\section{Introduction}

In recent times, graphene (Gr) filled polyaniline (PANI) nano-composites have grabbed a distinct importance scholastically and scientifically due to their salient features like stability, anti-corrosive property, simple acid/ base doping/de-doping, being light-weight, and are also quite inexpensive for composite preparation. They exhibit drastic changes in their properties physically based on the state of protonation and method of synthesis.

Most often, PANI has been exploited with carbonaceous materials to form composites in numerous technological applications such as sensors, electrodes for super capacitors and in nano electronics. Graphene is one such material which has facinated significant attention since its discovery due to its extremely interesting properties and extensive applications. Graphene is a monolayer sheet with carbon atoms organized in non-spatial arrangement of 2D honeycomb lattice with bond length $(C-C)$ of $0.142 \mathrm{~nm}$, particle size of $25 \mu \mathrm{m}$ and surface area $120-150 \mathrm{~m}^{2} / \mathrm{g}$. Graphene is a real sensational substance and has the prospective to craft disruptive technologies. A broad range of extraordinary features has been reported for graphene, ever since it was isolated in 2004 [1]. The

$\triangle$ Apsar Pasha, apsarpasha1982@gmail.com | ${ }^{1}$ Department of Physics, PES University, Bengaluru, Karnataka 560100, India. ${ }^{2}$ Department of Physics, Ghousia College of Engineering, Ramanagaram, Karnataka 562159, India. ${ }^{3}$ Department of Physics, Faculty of Science, University of Tabuk, Tabuk 71491, Kingdom of Saudi Arabia. ${ }^{4}$ Renewable Energy Laboratory, Nanotechnology Research Unit, Faculty of Science, University of Tabuk, Tabuk 71491, Kingdom of Saudi Arabia. ${ }^{5}$ Department of Mechanical Engineering, Ghousia College of Engineering, Ramanagaram, Karnataka 562159, India. 
important properties being having thermal conductivity of $\sim 5 \times 10^{3} \mathrm{~W} \mathrm{~m}^{-1} \mathrm{~K}^{-1}$, the higher electron mobility about $200,000 \mathrm{~cm}^{2} \mathrm{~V}^{-1} \mathrm{~s}^{-1}$ with electron densities in the order of $\sim 2 \times 10^{11} \mathrm{~cm}^{-2}$. The fact that all of these exceptional properties being originated from a single material has stimulated greater interest in $\mathrm{Gr}$ and has attracted researchers to use it in numerous scientific and technological applications such as field effect transistors, field emission devices, solar cells, actuators, batteries and in super capacitors [2-8]. The combination of PANI with Gr is intentive not only to fortify conducting polymers but also to produce interesting electronic properties on the basis of morphological modification or electronic interaction between the two materials. There are various methods used to produce PANI/graphite composites, one general procedure usually employed is in situ polymerization. This method is being employed to generate composites with non-covalent bonds where the Gr filler and PANI interact via comparatively weak dispersive forces which can be regarded as $\pi-\pi$ interaction between the quinoid rings of PANI and $\pi$-bond of graphite lattice [9-12].

In the present investigation, the authors report the effect of incorporating graphene on morphology, transport properties and Hall-effect of PANI. The prepared PANI/ Gr composites were examined through micro Raman spectroscopy, scanning electron microscopy (SEM), X-ray diffraction (XRD) and Fourier transform infrared spectroscopy (FTIR) for morphology and structural investigation. Detailed transport properties of pure PANI and PANI/Gr composites were investigated through DC/AC conductivities and dielectric behaviors. Through these studies, a fivefold increase in the conductivity of pure PANI was observed due to the addition of $\mathrm{Gr}$ in various weight percentages. The Hall-effect measurements were carried out in the prepared nanocomposites to understand the electronic properties in depth. In recent year, conducting polymer-graphene based nanomaterials composites have been used as new class of materials due to their improved properties compare to pure conducting polymers. It is impossible to develop opto-electronic devices by using graphene alone. In this work we have introduced graphene nano particles in PANI matrix to enhance electrical conductivity of the composites. Further, the Hall voltage was determined to be negative, indicating that the composite prepared herein behaves like a n-type semiconductor. Due to modified morphology and excellent electrical properties, the prepared PANI/Gr nanocomposites can be employed as a useful material in many electro-electrical applications.

\section{Experimental details}

\subsection{Materials used}

All the chemicals required such as the monomers of aniline, hydrochloric acid $(\mathrm{HCl})$, Ammonium peroxydisulphate (APS), Graphene powder and silver paste of research grade are procured from Sigma Aldrich (India) with a purity about 99.98\%.

\subsection{PANI synthesis}

PANI was synthesized through following in situ chemical oxidative polymerization procedure. The polymerization process was performed in a round bottom flask comprising of one molar of aniline and hydrochloric acid was dissolved in distilled water to achieve aniline hydrochloride. Around $100 \mathrm{ml}$ of $0.1 \mathrm{M}$ APS was mixed drop wise to the aniline hydrochloride solution rotated about $3 \mathrm{~h}$ at room temperature to start the polymerization process. The experiment was done and maintaining a very low temperature condition of $0-5{ }^{\circ} \mathrm{C}$. After this process the product was kept over ice bath over a period of 2 days, later this solution was double filtered using Whatmann's filter paper of pore size $0.5 \mu \mathrm{m}$ and precipitation of PANI was collected and exposed for washing in de-ionized water media. Ultimately, the collected PANI precipitate was subjected to vacuum drying [13].

\subsection{Synthesis of PANI/Gr composites}

Synthesis of PANI/Gr composites has also been carried out by following in situ polymerization technique. The $1 \mathrm{M}$ aniline solution and $1 \mathrm{M} \mathrm{HCl}$ were added to a flask and were dissolved in a pure water to get aniline hydrochloride. Gr was added to the solution and was repeatedly rotated for thirty minutes to obtain a uniform dispersion of $\mathrm{Gr}$ in aniline hydrochloride solution. To initiate polymerization of aniline, $0.1 \mathrm{M}$ of APS was mixed drop wise slowly to the above mixture with regular rotation about a period of $3 \mathrm{~h}$ keeping the temperature about $0-5{ }^{\circ} \mathrm{C}$. After 1 day, the solution of PANI/Gr was filtered and collected the precipitation of this solution, later washed in de-ionized water to get purity and finally this precipitation was dried in dynamic oven. In this method pure PANI and PANI doped with $\mathrm{Gr}$ with variable weight ratios such as 5, 10, 15, 20 and 25 wt $\%$ nanocomposites were prepared.

\section{Characterization details}

The surface morphology, different functional groups and structural confirmation of the synthesized pure PANI and PANI/Gr nanocomposites were investigated 
by employing various characterization methods such as SEM, XRD, FTIR and Raman spectroscopy. The FTIR spectra are reported in $\mathrm{KBr}$ medium employing a Perkin Elmer spectrometer (Model-Liantrisant CF728YWUnited Kingdom). The outor morphology in the prepared composites samples are studied applying ZEISS Ultra 60-SEM (Japan). The different functional groups present in pure PANI and PANI doped Gr nanocomposites were recorded by employing XPERT-3 X-ray diffractometer (Germany) with scan range $10^{\circ}-80^{\circ}$ upon scan rate of $0.03 \%$ s. The DC electrical conductivity of pure PANI and $\mathrm{PANI} / \mathrm{Gr}$ composites pressed in the form of pellets and examined using a indigenous DC conductivity setup which is comprised of Keithley 2182A NanoVoltmeter, 6221 DC and AC Current source and a furnace to hold the sample between the temperatures $60-200{ }^{\circ} \mathrm{C}$. The AC electrical conductivity measurements and dielectric attributes of the prepared composites were performed at room temperature by using four probes techniques using impedance analyzer-Wayne Kerr 6500B (London) in the frequency range of $100 \mathrm{~Hz}-5 \mathrm{MHz}$. The mechanical properties such as tensile stress and tensile strain of the prepared composites were carried out by using universal testing machine (UTM) as per the E-8M ASTM standard. By applying micrometer per minute displacement over composites to measure accurate tensile stress and tensile strain of the prepared composites.

\section{Results and discussion}

\subsection{Scanning electron microscope}

The SEM micrographs for pure PANI, graphene and PANI doped with 15 wt\% of graphene composite are shown in Fig. 1a-c. These micrographs obtained were investigated to understand the effect of incorporating Gr nanomaterials into PANI matrix. The SEM micrograph of pure PANI as shown in Fig. 1a represents granular agglomerated morphology. The exterior morphology of pure PANI represents, the grains are like cluster and shows a granular exterior morphology which is commonly seen in PANI prepared by the chemical oxidation method. In situ method is employed in the preparation of nanocomposites of PANI and $\mathrm{Gr}$ to yield highly aggregated and well embedded filler particles in a host matrix which is being revealed in the SEM micrographs. The composites prepared by the in situ method are highly aggregated and the morphology reveals that the graphene particles are well embedded in PANI matrix. The surface morphology of pure graphene is represented in Fig. $1 \mathrm{~b}$, it is clearly indicted that the grains are well resolved and grains are uniformly distributed. The surface morphology of PANI-Gr composites totally changed into clusters for the $15 \mathrm{wt} \%$ composite is shown in Fig. 1c. Also in this SEM micrograph the bulged structures of grains indicate the dispersion of graphene in PANI. The graphene particles one can observe a transition from
Fig. 1 SEM micrograph of a pure PANI, $\mathbf{b}$ pure graphene, c PANI doped with $15 \mathrm{wt} \%$ of graphene
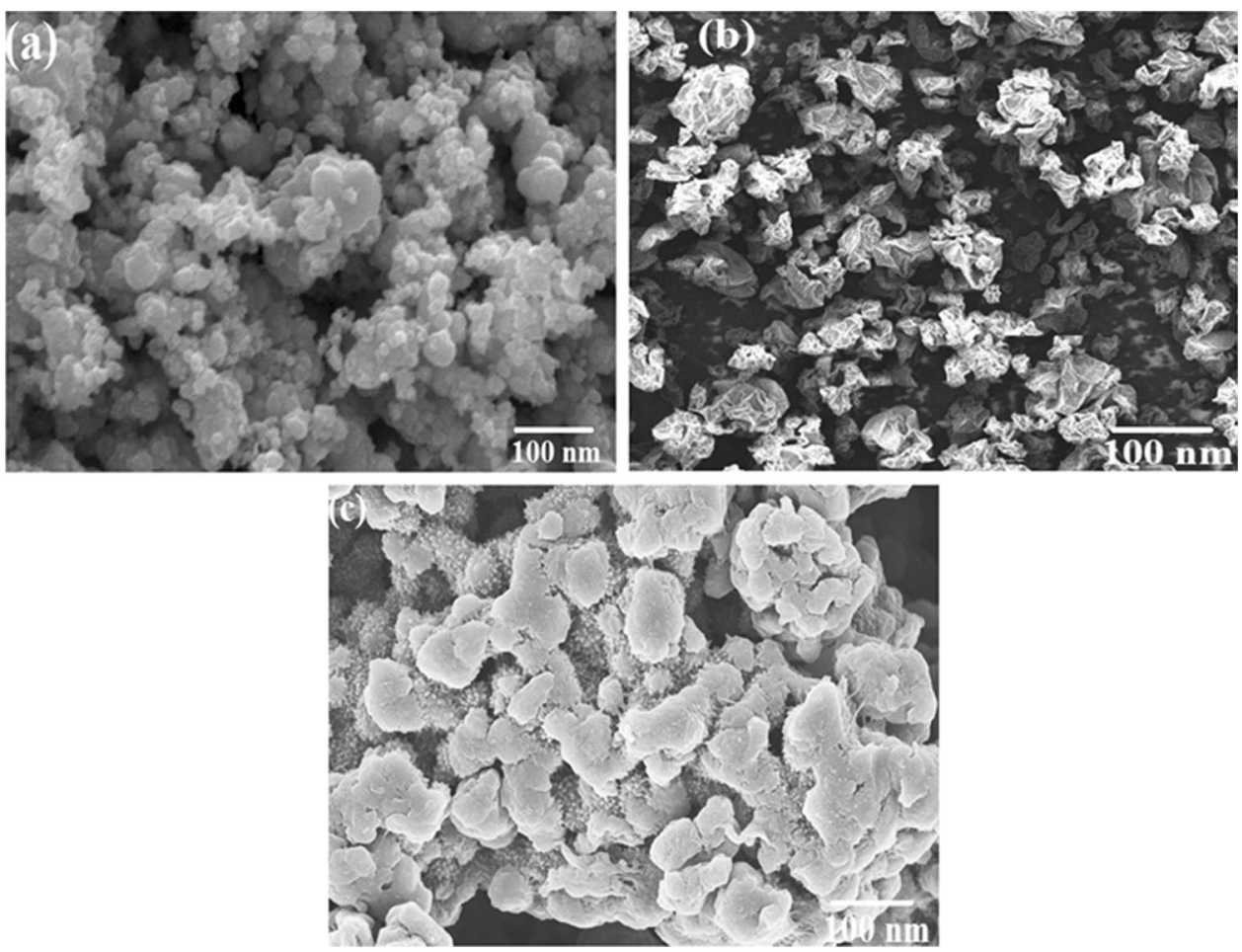

SN Applied Sciences A SPRINGER NATURE journal 
spherical to flake like layered morphology. The formation of flaked layer has increased in the composite structure with an increase in the concentration of graphene particles. It can be observed that in this micrograph the grains are well resolved and highly clustered morphology and this type of morphology helps for the charge carriers for easy transportation to the favourable sites in the polymer chain which results improved conductivity in PANI-Gr composites $[14,15]$.

\subsection{FTIR analysis}

Figure 2 shows the FTIR spectra of pure PANI, pure $\mathrm{Gr}$ and $15 \mathrm{wt} \%$ of PANI/Gr nanocomposite. The spectrum of pure PANI exhibits specific prominent peaks at $3480 \mathrm{~cm}^{-1}, 2420 \mathrm{~cm}^{-1}, 1390 \mathrm{~cm}^{-1}$ and $540 \mathrm{~cm}^{-1}$. The peak at $3480 \mathrm{~cm}^{-1}$ corresponds to the $\mathrm{O}-\mathrm{H}$ stretching vibration due to the existence of moisture in the prepared composites. The peak at $2420 \mathrm{~cm}^{-1}$ is due to asymmetric $\mathrm{N}-\mathrm{H}$ out of the plane stretching vibrations. The peak at $1390 \mathrm{~cm}^{-1}$ indicates the presence of $\mathrm{C}=\mathrm{C}$ benzoid ring and the peak at $540 \mathrm{~cm}^{-1}$ is designated to $\mathrm{C}-\mathrm{H}$ out of plane bending mode. The other peaks in the spectra of pure graphene at $950 \mathrm{~cm}^{-1}$ corresponds to $\mathrm{C}=\mathrm{O}$ vibration of acid chloride carbonyl derivative. The peaks at $1175 \mathrm{~cm}^{-1}$ and $1126 \mathrm{~cm}^{-1}$ corresponds to the stretching of sulphate that overlap with vibrations of epoxy groups. The obtained IR spectra of the synthesized nanocomposites for $15 \mathrm{wt} \%$ of PANI/Gr and pure Gr specify that the nanocomposite retains the significant major peaks of PANI, with a substantial rise in the intensity. The rise in the intensity of composite with a minute change in the frequencies of the nanocomposite theorizes a weak Vander Waal's interaction amongst PANI and $\mathrm{Gr}$ in the nanocomposite $[16,17]$.

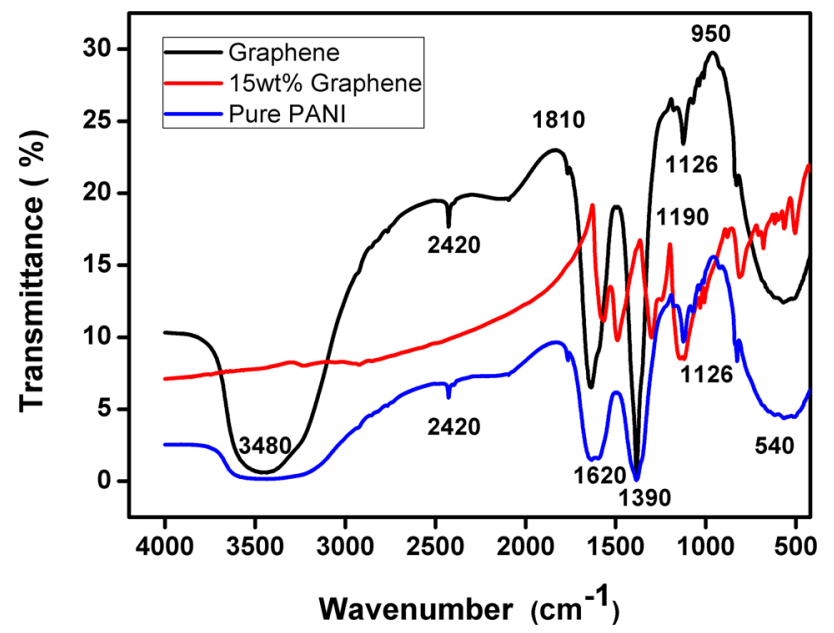

Fig. 2 FTIR spectra of PANI and PANI/graphene nanocomposites

\subsection{Raman spectroscopy}

The spectrum representing micro Raman spectroscopy analytical technique performed on the synthesized PANI/ Gr composites to characterize the structure and composition of the samples is shown in Fig. 3. Two important distinguishable peaks at 1350 and $1600 \mathrm{~cm}^{-1}$ are being noticed in the Raman spectra of PANI/Gr composite, which correspond to the $D$ band (is related to the presence of defects in the $s p^{2}$ crystal lattice, shows the pure graphene sheets) and $\mathrm{G}$ band (is associated to $s p^{2}$ carbon atoms chains spread in the complete polymer) respectively [18-20]. Also, the peaks at 2000, 2100, $2200 \mathrm{~cm}^{-1}$ are observed in the composite, the other peaks at smaller wavenumbers are not apparent due to they are too feeble or might be overlapped. The overlapping of the peaks $\left(1320,1480,1570\right.$ and $\left.1650 \mathrm{~cm}^{-1}\right)$ emerging from the graphene sheet arrive in PANI/Gr composite contributes to large $D$ and $G$ bands positioned at 1350 and $1600 \mathrm{~cm}^{-1}$, and its intensity increasing with enhancing the $\mathrm{Gr}$ concentrations in PANI. The main interaction among the PANI matrix and variable weight percentage of $\mathrm{Gr}$ was shown in the fitted with five Lorentzian curves. Three Lorentzian curves centered at 1610,1580 and $1340 \mathrm{~cm}^{-1}$ were investigated. The Raman spectra band at $1580 \mathrm{~cm}^{-1}$ is assigned to the $\mathrm{C}=\mathrm{C}$ stretching vibration in the quinonoid ring which is marginally shifted to $1590 \mathrm{~cm}^{-1}$ in the composite. Therefore, the peaks at 1340 and $1610 \mathrm{~cm}^{-1}$ correspond to $\mathrm{C}-\mathrm{N}$ vibration of delocalized polaronic structure and the $\mathrm{C}-\mathrm{C}$ stretching of the benzenoid ring respectively retaining the same position but with increased intensities. This could be interpreted as a deprotonation of amimine nitrogen atoms in PANI [21]. In the Raman spectra, the $I_{D} / I_{G}$ ratios of pure PANI and PANI doped graphene $(5,10,15,20,25 \%)$ composites is found to be $0.17,0.23,0.36,1.22,0.54$ and

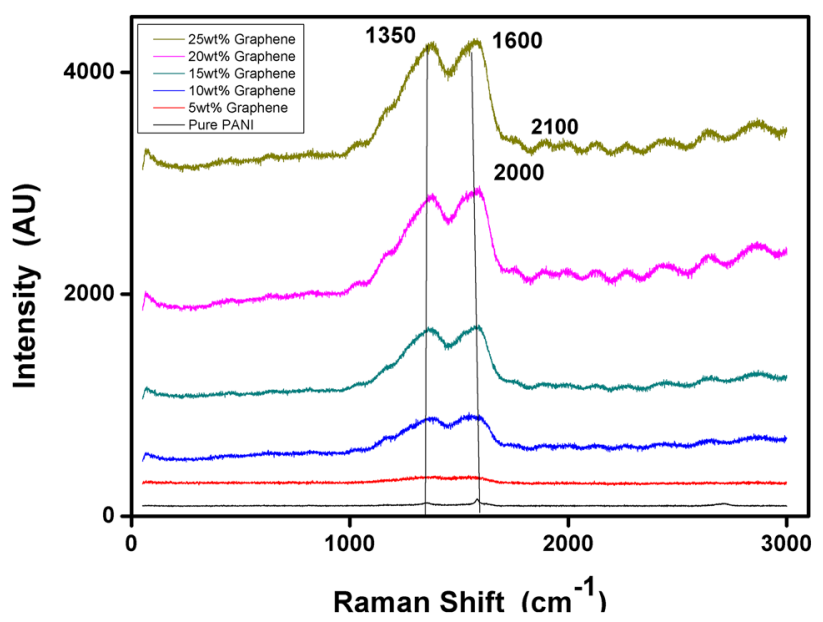

Fig. 3 Raman spectra of PANI and PANI/graphene nanocomposites 
0.21 respectively. When comparing the $\mathrm{I}_{\mathrm{D}} / \mathrm{I}_{\mathrm{G}}$ ratio in the Raman spectra, we can see that the shorter and broader the $D$ and $G$ bands are the higher the electrical conductivity. When compared to the $I_{D} / I_{G}$ ratio of the pure PANI and PANI doped graphene nano composites behaviour of increasing $\mathrm{I}_{\mathrm{D}} / \mathrm{I}_{\mathrm{G}}$ ratio is similar after reduction with the difference being in the absolute values, rather than the relative shift. Among the prepared the composites $15 \mathrm{wt} \%$ composites shows higher value $I_{D} / I_{G}$ ratio in the order of 1.22 reaches a higher electrical conductivity, compared with other prepared nanocomposites, attribute due to more $\pi$-electrons conjugation in the polymer backbone which results improved conductivity was achieved. As in our work, $15 \mathrm{wt} \%$ composites has the highest electrical conductivity with highest $\mathrm{I}_{\mathrm{D}} / \mathrm{I}_{\mathrm{G}}$ ratio.

\subsection{XRD analysis}

The XRD diffractograms of PANI and PANI/Gr nanocomposites are shown in Fig. 4. The diffractogram of PANI exhibits a semi-crystalline peak at $26^{\circ}$ which is the major distinctive peak of PANI [22]. The broad peak of PANI is superimposed by the diffraction peak of $\mathrm{Gr}$ and a highly pronounced peak of PANI doped $\mathrm{Gr}$ is observed at $27^{\circ}$ in the $15 \mathrm{wt} \%$ composite diffractogram whose height increases proportionally to the weight percentage of $\mathrm{Gr}$ in the polymer matrix. The evidence of the $\mathrm{Gr}$ peaks at $27^{\circ}, 45^{\circ}$ and $54^{\circ}$ in the composite confirms the formation and modification of the composite. But the XRD pattern of composites shows more sharp peaks in compare to pure PANI peaks suggested that improved crystallinity in the composites in compare to pure PANI. The obtain XRD pattern is clearly indicated, the composites having improved $\pi$-electron delocalization which may be reason to improve the electrical conductivity in PANI/Gr nanocomposites.

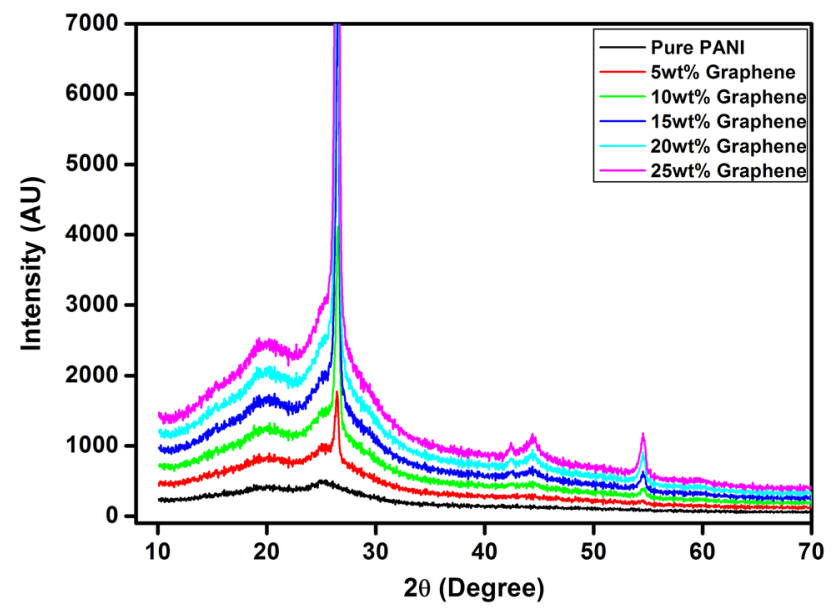

Fig. 4 XRD patterns of PANI and PANI/graphene nanocomposites

\section{Transport properties}

The total contribution to electrical conductivity in conducting polymers system is divided into frequency independent DC part and frequency dependent AC part. To measure the electrical conductivity in conducting polymers generally used either two probes and four probes methods used to investigate the electrical properties of the materials. The conductivities of PANI and PANI/Gr composites with $5,10,15,20,25 \mathrm{wt} \%$ were measured using a indigenous experimental setup.

Figure 5 represents the variation in DC conductivity with temperature for various wt $\%$ of $\mathrm{Gr}$ in PANI. From the plot, it can be noticed that the conductivity values are almost constant up to a temperature of $115^{\circ} \mathrm{C}$ in all the samples of the composite, whereas the conductivity was observed to be constant up to $160^{\circ} \mathrm{C}$ in case of pure PANI. At higher temperatures, a substantial raise in conductivity has been recorded which is because of the enhanced efficiency of charge carrier transfer between $\mathrm{Gr}$ and PANI with raise in temperature. The thermal curling influences the arrangement of polymer chains in the network and as a result the conjugation length is expected to increase, which subsequently results in improved conductivity. Also, the rearrangement of molecules upon heating makes them favorable for electron delocalization in the lattice [23]. The clusters formed due to the inclusion of Gr of different weight percentages pave a path for the formation of polarons and bipolarons and at higher temperatures, these charge carriers get assistance for hopping from thermal stimulation as per variable range hopping (VRH) theory. As the charge carriers gain thermal energy, they

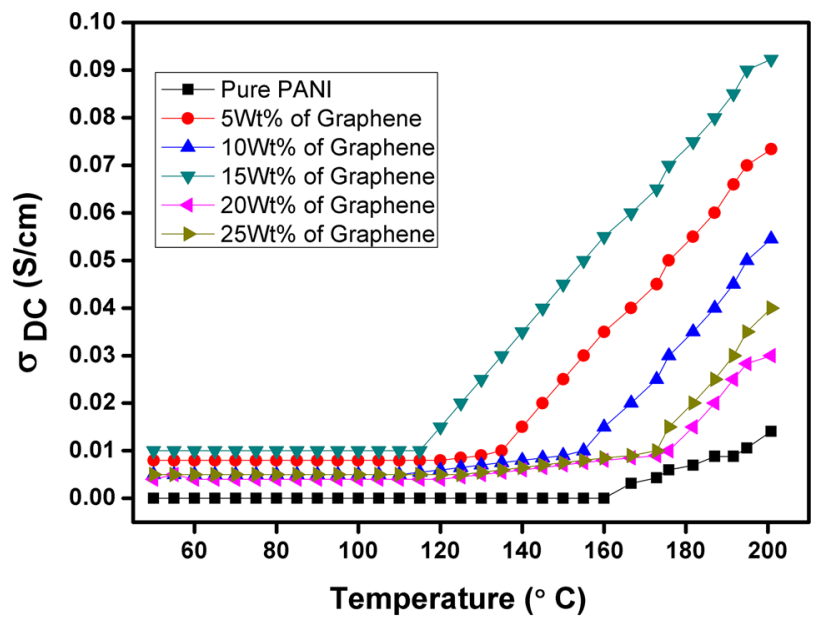

Fig. 5 Variation of temperature dependent conductivity with respect to temperature in PANI and PANI/graphene nanocomposites 
move with intensified velocity resulting in enhanced conductivity due to improved charge carrier mobility. Among all the composites, higher conductivity is being recorded for $15 \mathrm{wt} \%$ of PANI/Gr nanocomposite which is regarded as a percolation threshold and is less for other composites as depicted in Fig. 6. The $15 \mathrm{wt} \%$ PANI/Gr sample exhibits maximum conductivity value of $0.0923 \mathrm{~S} / \mathrm{cm}$ and the fall in the conductivity value for the other composites is because of the obstruction caused to the charge carriers making their flow difficult with increase in the wt $\%$ of $\mathrm{Gr}$ in PANI which is beyond the percolation limit. The decrease of conductivity after percolation limit $(20,25 \mathrm{wt} \%)$ composites, is might be due to reducing the $\pi$-electron conjugation in the polymer chain, which results more distructions for transportation of charge carriers (polarons and bipolarons) due to poor dispersion of graphene in PANI. Further, in microscopic level the decrease in conductivity is observed which may be attributed due to the distribution of graphene particles of larger grain size which are partially blocking the hopping of charge carriers in the polymer matrix.

Figure 7 illustrates the plot representing the change of AC conductivity in PANI and PANI/Gr nanocomposites with respect to frequency. A modification in the electrical response was observed with the inclusion of graphene in PANI due to the formation of agglomerates, cross links and conductive paths which are favorable for electrical conduction in these composites. It can be observed from the plot that the values of AC conductivity increase with applied frequency implying a specific nature in the disordered systems, where the mechanism of hopping overtakes the tunneling of charge carriers. The plots reflect a frequency independent plateau region followed by a high frequency dispersed region in PANI and PANI/Gr

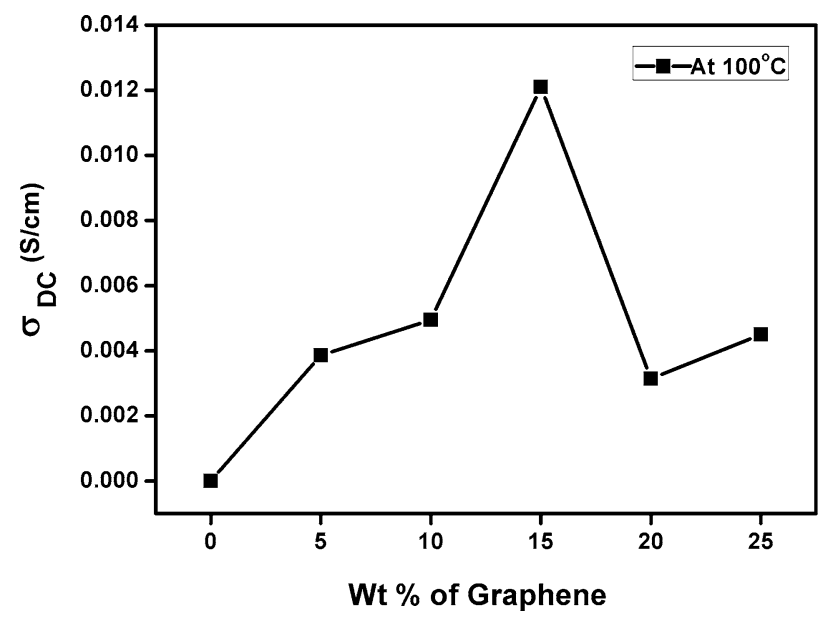

Fig. 6 Variation of temperature dependent conductivity in PANI and PANI/graphene nanocomposites at $100^{\circ} \mathrm{C}$

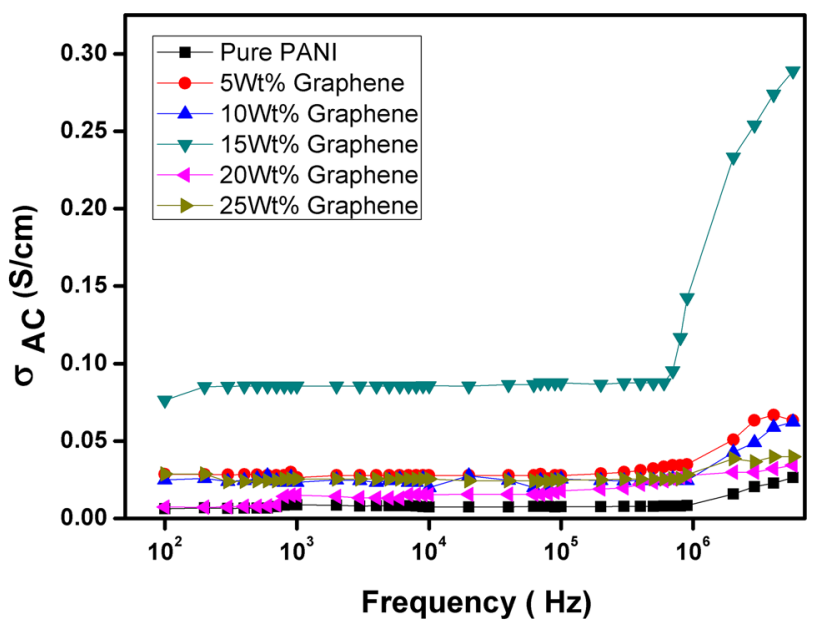

Fig. 7 Variation of frequency dependent conductivity with respect to frequency in PANI and PANI/graphene nanocomposites

nanocomposites. The evidence that, the AC conductivity consist of frequency dependent part and independent part above the threshold frequency indicates a traverse from DC to AC conductivity [24]. It can be observed that up to $10^{6} \mathrm{~Hz}$ all the composite including PANI exhibited almost a constant value where AC conduction overtakes and notable change in conductivity values of the composites is being observed above $10^{6} \mathrm{~Hz}$ frequency. The variation in AC conductivity with frequency towards higher frequencies is accord with the universal power law [25]. The content of $\mathrm{Gr}$ was found to affect considerably the conducting ability in the prepared composites. Among the prepared composites, 15 wt $\%$ of PANI/Gr nanocomposite shows highest conductivity as shown in Fig. 8. Such a high conductivity value for the composites at percolation threshold is an evidence indicating particle-particle interface. The other samples are not as highly conducting as $15 \mathrm{wt} \% \mathrm{PANI} / \mathrm{Gr}$ sample due to discontinuous charge delocalization process as a result of chain imperfections and more disorderliness within the system.

\subsection{Dielectric properties}

Figures 9 and 10 shows the variation in real $\left(\varepsilon^{\prime}\right)$ and imaginary $\left(\varepsilon^{\prime \prime}\right)$ parts of the dielectric constant for various wt\% of Gr in PANI respectively. The permittivity plots are found to be effectively dispersed towards the lower frequency range which remains constant with frequency beyond $10^{3} \mathrm{~Hz}$. The permittivity behavior depicted in the plots is mainly due to dipole polarization mechanism occurring in these conducting polymer systems [26]. Larger values of permittivity at the low frequency side are due to building-up of greater percentage of charge carriers at the interface of grain boundaries which included 


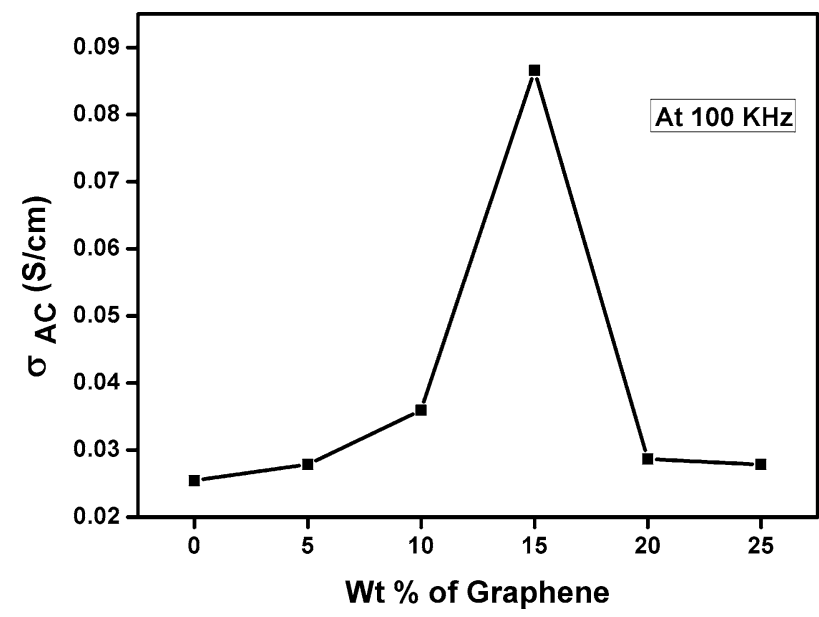

Fig. 8 Variation of frequency dependent conductivity in PANI and $\mathrm{PANI} /$ graphene nanocomposites at $100 \mathrm{kHz}$

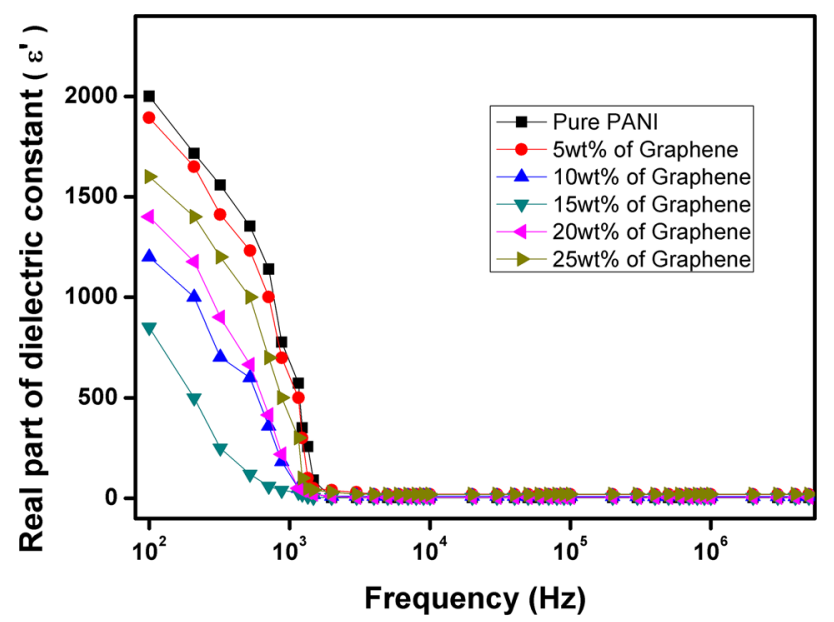

Fig. 9 Variation of the real part of dielectric constant with respect to frequency for PANI and PANI/graphene nanocomposites

in polarization mechanism. The interaction between $\mathrm{Gr}$ particles and chains of PANI hinder the motion of dipoles on account of which dielectric constant gradually drop at greater frequencies. In all the prepared composites, it has been seen that the dielectric constant value is lower in the case of 15 wt $\%$ of $\mathrm{Gr}$ doped PANI composites followed by other prepared composites. It can also be noticed that the value of dielectric constant diminished at $10^{3} \mathrm{~Hz}$ frequency range due to non-existence of dipole relaxation in the nanocomposites. The imaginary part of permittivity actually represents the loss. The energy loss takes place when a particular type of polarization ceases due to applied frequency and the dipoles become inefficient to follow the changing field. For a frequency greater than $10^{3} \mathrm{~Hz}$, which is greater than the relaxation frequency of space charge

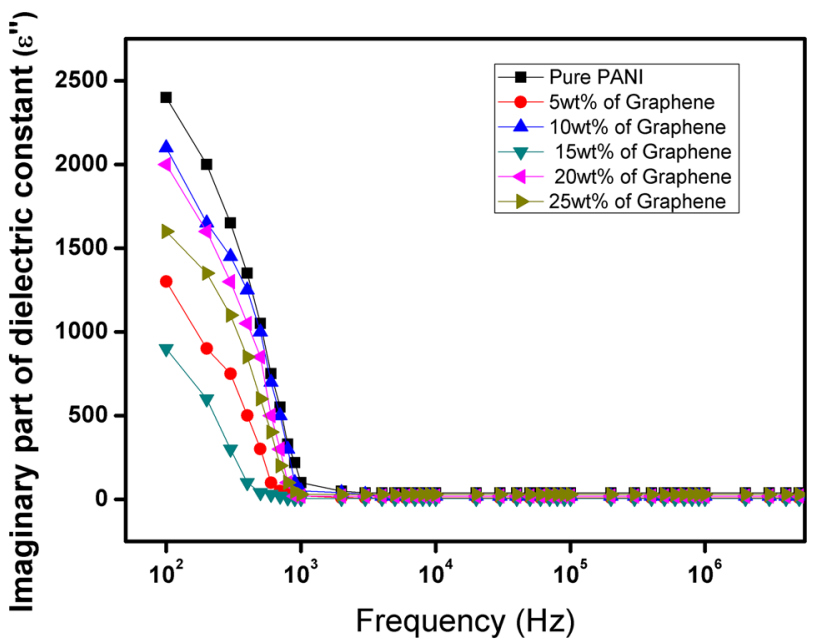

Fig. 10 Variation of the imaginary part of dielectric constant with respect to frequency for PANI and PANI/graphene nanocomposites

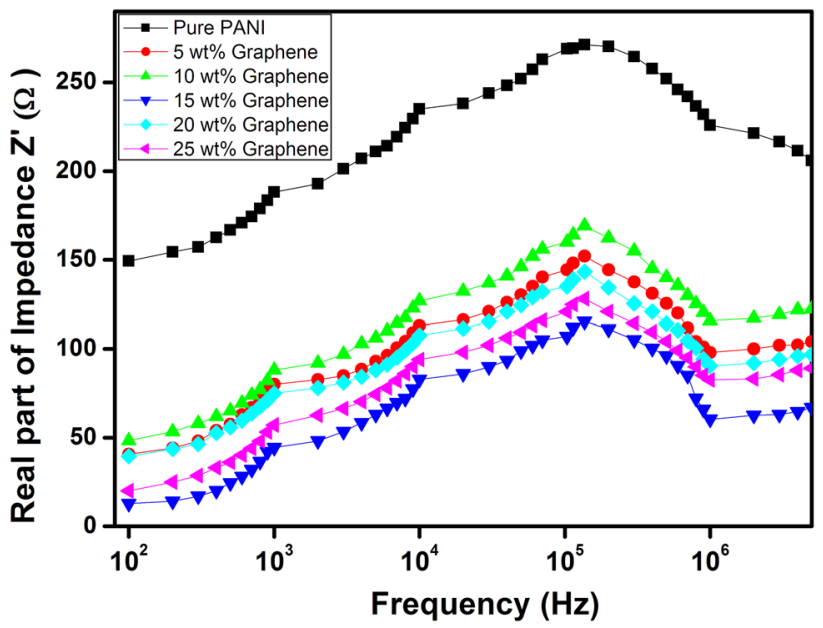

Fig. 11 Variation of the real part of impedance with respect to frequency for PANI and PANI/graphene nanocomposites

polarization, the switching action of the dipoles cannot keep in step with that of the changing field and hence the polarization mechanism is halted resulting in a decrease in the value of imaginary dielectric constant. Amid all the composite samples, the real part and imaginary part of dielectric constant for $15 \mathrm{wt} \%$ of Gr in PANI is very much less compared to pure PANI.

The variation in the real and imaginary $\left(Z^{\prime}\right.$ and $\left.Z^{\prime \prime}\right)$ part of impedance with respect to applied frequency for various wt\% of Gr in PANI composites were depicted in Figs. 11 and 12 respectively. From the plots, it is observed that the values of impedance decreases at higher frequencies region and the variation of impedance is depends on the filler concentration in PANI/Gr composite. In the present multi-phase composite samples, three different processes 
involving dielectric dispersion, charge carrier accumulation and the union of both charge carriers and dielectric dispersion are expected to occur within the estimated frequency range. The relaxation peaks in both real and imaginary part of impedance is due to existence of space charge relaxation. Therefore, the electrical conductivity is inversely proportional to impedance [26]. In the real part of impedance, it can be seen that with rise in frequency up to a particular range, $Z^{\prime}$ is observed to decrease and further it remains unaffected. This phenomenon may be a consequence of grain resistance. Added to that towards low frequency region, there is a higher dissipation due to the collective polarization mechanism of dipoles. Amid all the prepared composites, $15 \mathrm{wt} \% \mathrm{PANI} / \mathrm{Gr}$ nanocomposite gives the least impedance value, signifying that Gr particles at percolation, generates a greater number of free moving charge carriers and further transportation of these charge carriers for favorable sites is because of the fine grain periphery.

Figure 13 depicts the Cole-Cole plot, which is acquired by plotting real and imaginary part of impedance for the PANI/Gr. It is clearly seen from the figure, that the $15 \mathrm{wt} \%$ PANI/Gr exhibits least impedance compared to all other composites. The values of impedance depend on the total resistance in the circuit which is the summation of grain resistance and bulk resistance. The establishment of semicircular arcs signifies the dielectric response where a resistor and capacitor are in parallel also the drop in the arc area signifies the raise in the conductivity. Added to that the generation of two distinct semicircular arcs in series reveals the existence of various components in the composite offering the resistance.

Figure 14 shows the variation of quality factor for variable wt\% of Gr in PANI with frequency. In this plot, it can

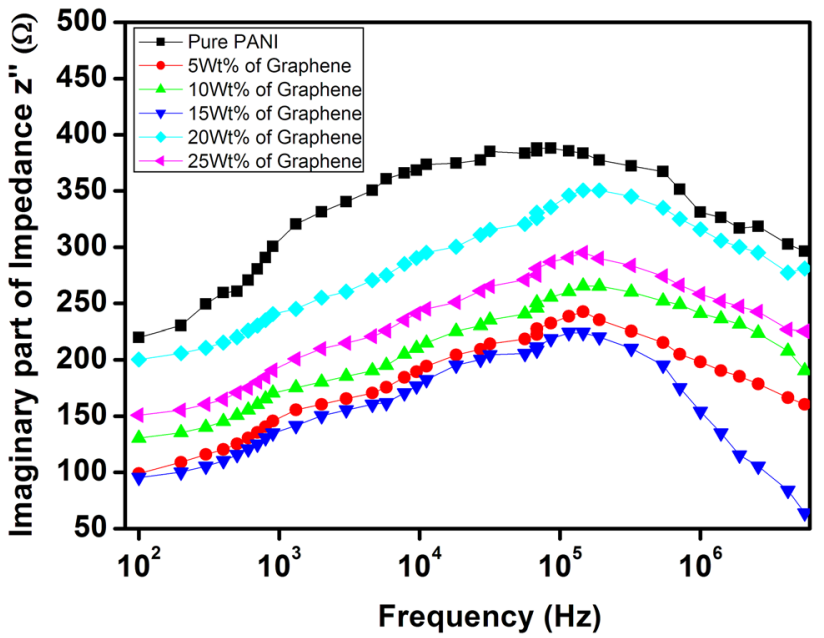

Fig. 12 Variation of the imaginary part of impedance with respect to frequency for PANI and PANI/graphene nanocomposites

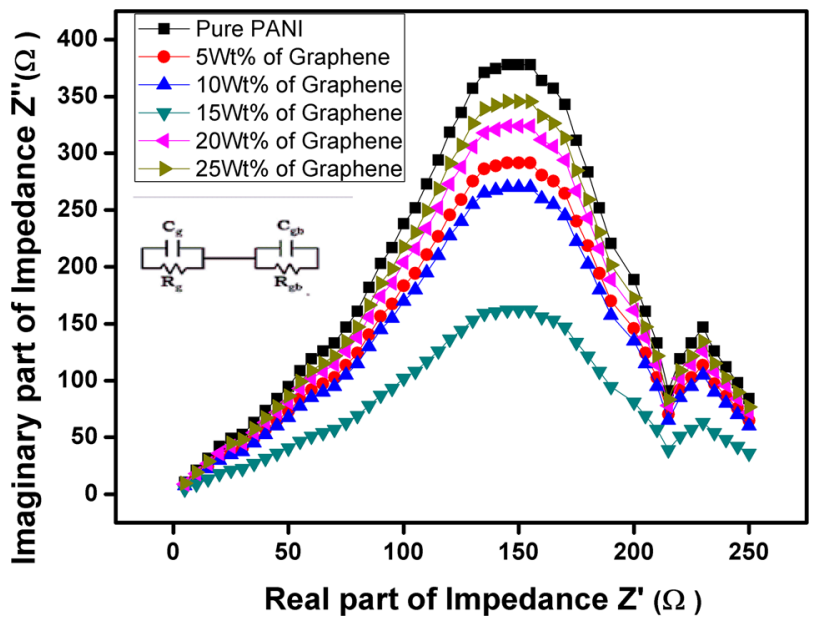

Fig. 13 Cole-Cole plot of PANI and PANI/graphene nanocomposites

be observed that PANI has the least quality factor compared to the other prepared composite. The presence of Gr particles in the polymer chain facilitates to lower the oscillations which results in the enhancement of quality factor. Among all the prepared samples, $15 \mathrm{wt} \%$ PANI/Gr nanocomposite shows the highest quality factor of 0.0006 . Compared to extreme levels of frequency, maximum value of quality factor is observed in the frequency range of $10^{4}-10^{5} \mathrm{~Hz}$. This may be due to the lower oscillations of the particles in this region, that is the oscillations of the system are almost damped, under the impact of external frequency and therefore such materials can be utilized in electronically stable devices.

Figure 15 represents the variation in tangent loss in case of all the composite samples of PANI/Gr. From this plots it

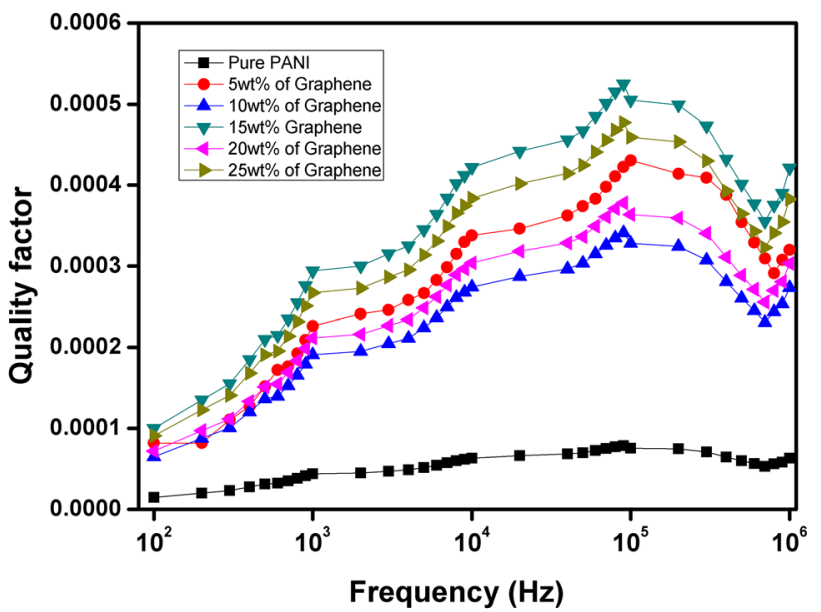

Fig. 14 Variation of quality factor for PANI and PANI/graphene nanocomposites 


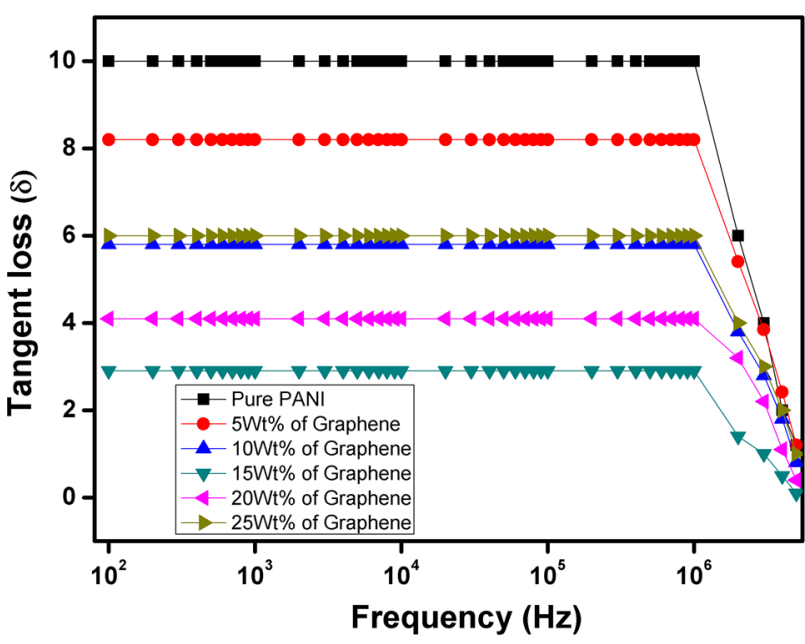

Fig. 15 Variation of tangent loss for PANI and PANI/graphene nanocomposites

is observed that the dielectric loss value is higher for PANI and the value remains constant up to $10^{6} \mathrm{~Hz}$ frequency in all the samples of the composite. However, it is recorded to be lower for $15 \mathrm{wt} \%$ composite sample and gradually decreases further towards higher frequencies. The dielectric loss values were observed to be reduced completely with the increase in frequency beyond $10^{6} \mathrm{~Hz}$ for all the composites. When the applied frequency becomes greater than the relaxation frequency of inter-facial polarization which is usually expected to occur in disordered heterogeneous materials, the switching action of the dipoles cannot keep in step with that of the changing field and they decline to response towards the applied field and result in an energy loss [27]. For frequency above the $10^{6} \mathrm{~Hz}$, the tangent loss was found to be significantly drop, which represents the easy charge transportation within the polymer chains $[28,29]$.

\section{Hall effect studies}

In contemporary circuits and semiconductor machineries, knowledge of the two important parameters $\mathrm{n}$ and $\mathrm{p}$ is compulsory. Presently in research laboratories and semiconductor industries, Hall effect measurements are considered to be one of the most commonly used characterization tools [30]. Moreover, the conducting polymer composites usually exhibit conductivity in semiconducting range and therefore are conducting semiconductors. Hall effect measurements are essential for semiconductor materials to distinguish between negative charges moving in one direction and positive charges moving in the opposite. Hall effect experimental results can be used to interpret the conductivity type, Hall voltage, mobility and carrier density parameters. In addition, measuring mobility of charge carriers is very much essential for designing efficient transistor switching and effective charge separation in photovoltaic devices [31].

In the present study, the PANI and PANI/Gr composite were prepared are placed in the specified position within the strong magnetic field $(0.36 \mathrm{~T})$. As the charge carriers are in motion, a magnetic field is developed and its magnetic field is distorted by the magnetic field. This magnetic force which disturbs the direction of flow of charges is called Lorentz force. Due to this distortion in the magnetic field of the charge carriers, the negative charged electrons are deflected to one side of the plate and positive charged holes to the other side, from which a potential difference is generated. This is called Hall voltage. The Hall effect studies were carried out using a specially designed Hall four probe with Hall effect set up called Vanderpauw method. Hall voltage was measured by applying $0.36 \mathrm{~T}$ of magnetic field and changing current through the samples in steps of $0.5 \mathrm{~mA}$ at room temperature. The graph plotted between Hall voltage and sample current is shown in Fig. 16. From the plots it can be inferred that among all the samples, the measured Hall voltage is greater in case of the composite samples than pure PANI. The observed result is also supported by the temperature dependent conductivity results of PANI and PANI/Gr composite. Hall voltage for $15 \mathrm{wt} \%$ PANI/Gr nanocomposite is 1.7 times, carrier concentration is 1.21 times and mobility is 9.15 times greater than that of pure PANI. From the Hall effect studies, it can also be interpreted that the conductivity and carrier mobility are linearly related, whereas the carrier mobility and carrier density are inversely proportional which is illustrated in Fig. 17. The Hall voltage was determined to be negative,

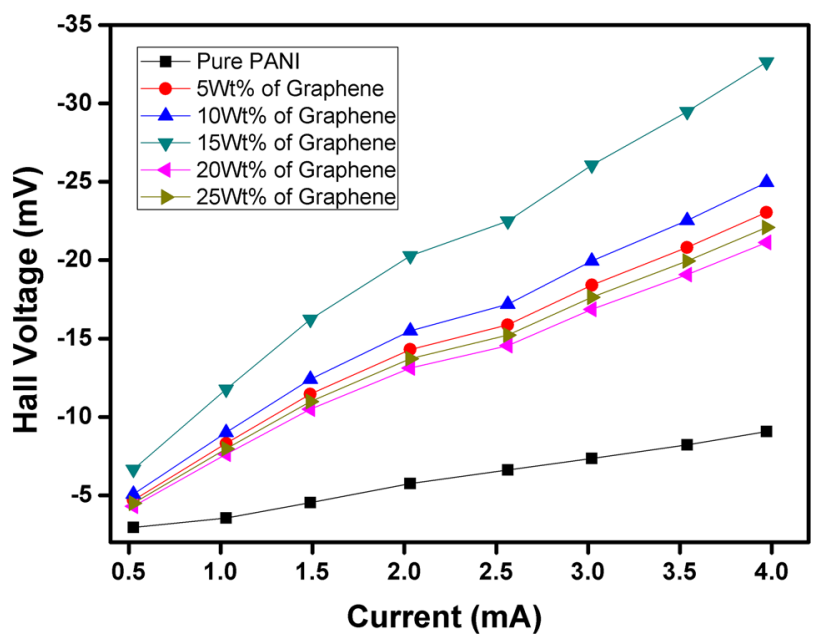

Fig. 16 Variation of Hall voltage with respect to sample current for PANI and PANI/graphene nanocomposites 


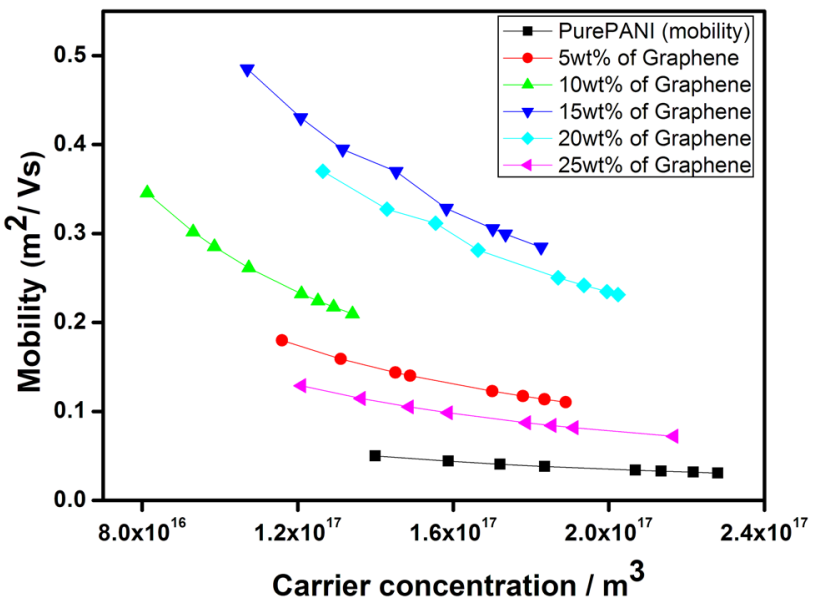

Fig. 17 Variation of number of charge carriers with respect to mobility for PANI and PANI/graphene nanocomposites

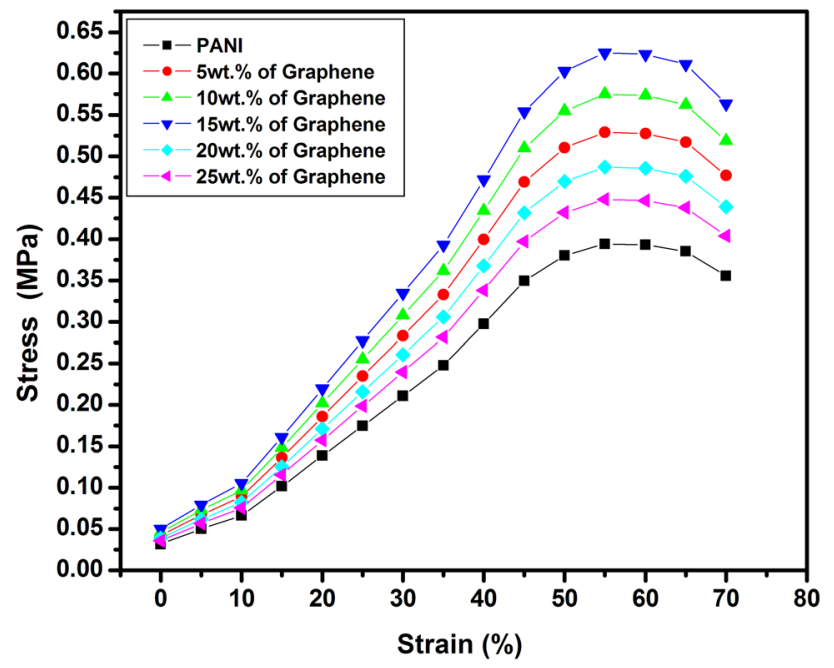

Fig. 18 Variation of tensile stress-strain behavior of PANI and PANI/ graphene nanocomposites

indicating that the composite prepared herein behaves like a n-type semiconductor.

\section{Mechanical properties}

The variation of tensile stress and tensile strain of pure PANI and PANI doped with different weight percentage of graphene nanocomposites were studied at room temperature is depicted in Fig. 18. It is observed that the tensile stress and tensile strain plots of the composites, the pure PANI shows lowest value of tensile stress $0.10 \mathrm{MPa}$ and PANI doped $15 \mathrm{wt} \%$ graphene shows $0.62 \mathrm{MPa}$. It is observed that, the tensile stress is linearly increases with applied percentage of tensile strain in all prepared composites and sudden decreases at $55 \%$ of tensile strain due to the composites undergoes crack due to the higher tensile strain. It is observed that in case of $15 \mathrm{wt} \% \mathrm{com}$ posites show higher tensile stress might be due to good compact morphology which results high rigidity in the composite this behavior can be correlated to SEM morphology. But, in the case pure PANI and PANI doped graphene $(5,10,20,25 \mathrm{wt} \%)$ composites the tensile stress is low due to the grains in the polymer matrix is not well interconnected each other, because of brittleness in the nanocomposite [32-34].

\section{Conclusion}

In this research work, the pure PANI and PANI/Gr nanocomposites were synthesized via in situ polymerization approach. The prepared composites were examined through FTIR, XRD, SEM and micro Raman spectroscopy, these experimental techniques provide an information about the surface morphology, interactions between the different functional groups, degree of crystallinity and structure confirmations in the prepared composites. The dispersion of graphene in PANI matrix which is significantly enhance the electrical properties in this materials composites due to harmonic interactions between the $\mathrm{Gr}$ and PANI matrix. The enhanced conformational changes after doping $\mathrm{Gr}$ in PANI matrix which lead to improved $A C$ and $D C$ electrical conductivities. Among the prepared composites the $15 \mathrm{wt} \%$ PANI/Gr composites shows higher electrical conductivity and improved Hall voltage due to enhancement in conjugation length in the polymer matrix and thermally assisted hopping of charge carriers. It is observed that the electrical conductivity was decreased in other weight percentage composites, but the percolation threshold was observed in the case of $15 \mathrm{wt} \%$ of $\mathrm{Gr}$ in PANI. The addition of graphene in PANI is greatly influenced on dielectric constant and dielectric loss in these polymer system. The Hall effect studies also have revealed that the Hall voltage and mobility of charge carriers is higher for 15 wt\% PANI/Gr composite. Further, the PANI and PANI doped with graphene composites were carriedout the mechanical properties such as tensile stress and tensile strain, this study it is found that $15 \mathrm{wt} \%$ composite is higher tensile stress. Based on the studies of these $\mathrm{Gr}$ treated PANI composites, they are exploited for possible applications towards the fabrication of electro-electric devices.

Acknowledgements The authors would like to thank the Management and Principal of PES University, Bangalore South Campus, for their cooperation and assistance to carry out this research work. 


\section{Compliance with ethical standards}

Conflict of interest The authors declare that they have no competing interests.

\section{References}

1. Novoselov KS, Geim AK, Morozov SV, Jiang D, Zhang Y, Dubonos SV (2004) Electric field effect in atomically. Thin Carbon Films Sci 306:666

2. Bolotin KI, Sikes K, Jiang Z, Klima M, Fudenberg G, Hone J, Kim P, Stormer HL (2008) Ultra high electron mobility in suspended graphene. Solid State Commun 146:351

3. Balandin AA, Ghosh Bao S, Calizo I, Teweldebrhan D, Miao F, Lau CN (2008) Superior thermal conductivity of single-layer graphene. Nano Lett 8(3):902

4. Bunch JS, Verbridge SS, Alden JS, Van der Zande AM, Parpia JM, Craighead HG, McEuen PL (2008) Impermeable atomic membranes from graphene sheets. Nano Lett 8(8):2458

5. DuX SI, Barker A, Andrei EY (2008) Approaching ballistic transport in suspended graphene. Nat Nanotechnol 3:491

6. Castro Neto AH, Guinea F, Peres NKS, Geim AK (2009) The electronic properties of graphene. Rev Mod Phys 81:109

7. Wang R (2017) Hydrothermal synthesis of nanostructured graphene/polyaniline composites as high-capacitance electrode materials for supercapacitors. Sci Rep 7:44562

8. Zhang K, Zhang LL, Zhao XS, Wu JS (2010) Graphene/polyaniline nanofiber composites as supercapacitor electrodes. Chem Mater 22:1392

9. Lee C, Wei X, Kysar JW, Hone J (2008) Measurement of the elastic properties and intrinsic strength of monolayer graphene. J Sci 321:385

10. Hirata M, Gotou T, Ohba M (2005) Thin-film particles of graphite oxide. 2: Preliminary studies for internal micro fabrication of single particle and carbonaceous electronic circuits. Carbon 43:503

11. Szabo T, Szeri A, Dekany I (2005) Composite graphitic nanolayers prepared by self-assembly between finely dispersed graphite oxide and a cationic polymer. Carbon 43:87

12. Mostafaei A, Nasirpouri F (2014) Epoxy/polyaniline-ZnO nanorods hybrid nanocomposite coatings: synthesis, characterization and corrosion protection performance of conducting paints. Prog Org Coat 77:146

13. Lakshmi M, Roy AS, Khasim S, Faisal M, Sajjan KC, Revanasiddappa M (2013) Dielectric property of $\mathrm{NiTiO}_{3}$ doped substituted ortho-chloropolyaniline composites. AIP Adv 3:112

14. Kumar R, Sahoo S, Joanni E, Singh RK, Yadav RM, Verma RK (2019) A review on synthesis of graphene, $\mathrm{h}-\mathrm{Bn}$ and $\mathrm{MoS}_{2}$ for energy storage applications: recent progress and perspectives. Nano Res 12(11):2655-2694

15. Kumar R, Sahoo S, Joanni E, Singh RK, Tan WK, Kar KK, Matsuda A (2019) Recent progress in the synthesis of graphene and derived materials fornext generation electrodes of high performance lithium ion batteries. Prog Energy Combust Sci 75:100786

16. Badi N, Khasim S, Roy AS (2016) Micro-Raman spectroscopy and effective conductivity studies of graphene nanoplatelets/polyaniline composites. J Mater Sci Mater Electron 27:624

17. Elnaggar EM, Kabel KI, Farag AA, Al-Gamal AG (2017) Comparative study on doping of polyaniline with graphene and multiwalled carbon nanotubes. J Nanostruct Chem 7:75
18. Eckmann A, Felten A, Verzhbitskiy I, Davey R, Casiraghi C (2013) Raman study on defective graphene: effect of the excitation energy, type, and amount of defects. Phys Rev B 88:035426

19. Tuinstra F, Koenig J (1970) Raman spectrum of graphite. J Chem Phys 53:1126

20. Lakshmi M, Khasim S, Roy AS, Parveen A, Al-Hartomy OA (2015) Synthesis, characterization, and dielectric studies of orthochloropolyaniline/graphite oxide composites. J Mater Res 30:2310-2318

21. Majumdar D (2016) Functionalized-graphene/polyaniline nanocomposites as proficient energy storage material: an overvie. Innov Energy Res 5(2):145

22. Majumdar D, Baskey M, Saha SK (2011) Epitaxial growth of crystalline polyaniline on reduced graphene oxide. Macromol Rapid Commun 32:1277

23. Khasim S, Lakshmi M, Johani H-A, Badi N, Roy AS (2018) Investigations on structural and electrical properties of polyanilinecadmium sulfide nanocomposite films for solid state electronics. Polym Compos 30:2310

24. Khasim S (2019) Polyaniline-graphene nanoplatelet composite films with improved conductivity for high performance $X$-band microwave shielding applications. Results Phys 12:1073

25. Mishra A, Choudhary SN, Prasad K, Choudhary RNP (2011) Complex impedance spectroscopic studies of $\mathrm{Ba}(\operatorname{Pr} 1 / 2 \mathrm{Ta} 1 / 2)$ $\mathrm{O}_{3}$ ceramic. Phys B 406:3279

26. Kyritsis A, Pissis P, Grammatikakis J (1995) Dielectric relaxation spectroscopy in poly(hydroxyethyl acrylates)/water hydrogels. J Polym Sci Part B Polym Phys 33:1737

27. Mott NF (1990) Metal-insulator transition, 2nd edn. Taylor \& Francis, London

28. Roy AS, Anilkumar KR, Ambika Prasad MVN (2011) Core-shell method of synthesis, characterizations, and ac conductivity studies of polyaniline/n- $\mathrm{TiO}_{2}$ composites. J Appl Polym Sci 121:675

29. Roy AS, Anilkumar KR, Ambika Prasad MVN (2012) Studies of AC conductivity and dielectric relaxation behavior of CdO-doped nanometric polyaniline. J Appl Polym Sci 123:1928

30. Bahrami A, Talib ZA, Yunus WMM, Behzad K, Abdi MM, Din FU (2012) Low temperature Hall effect investigation of conducting polymer-carbon nanotubes composite network. Int J Mol Sci 13:14917

31. Kasap S, Capper P (2017) Handbook of electronic and photonic materials. Springer, Berlin

32. Giripunje SM, Ghushe J (2013) Preparation, characterization and Hall effect study ofconducting polyaniline and polyaniline- $\mathrm{ZnO}$ nano composite. J Nano Res 24:122-132

33. Pomfret SJ, Adams PN, Comfort NP (2000) Electrical and mechanical properties of polyaniline fibres produced by a onestep wet spinning process. Polymer 41(6):2265-2269

34. Valentova H, Stejskal J (2010) Mechanical properties of polyaniline. Synth Met 160(7-8):832-834

Publisher's Note Springer Nature remains neutral with regard to jurisdictional claims in published maps and institutional affiliations. 